\title{
Witten-Veneziano Relation for the Schwinger Model
}

\author{
S.Azakov ${ }^{1 *}$, H.Joos ${ }^{2 \dagger}$ and A.Wipf $3 \ddagger$
}

October 24, 2018

${ }^{1}$ Inst. of Physics, Azerbaijan Academy of Sciences, H.Cavid ave.33, Baku 143, Azerbaijan

${ }^{2}$ DESY, Notkestrasse 85, D-22603 Hamburg, Germany

${ }^{3}$ Theor.-Phys. Institut, Universität Jena, Fröbelstieg 1, D-07743 Jena, Germany

\begin{abstract}
The Witten-Veneziano relation between the topological susceptibility of pure gauge theories without fermions and the main contribution of the complete theory and the corresponding formula of Seiler and Stamatescu with the so-called contact term are discussed for the Schwinger model on a circle. Using the (Euclidean) path integral and the canonical (Hamiltonian) approaches at finite temperatures we demonstrate that both formulae give the same result in the limit of infinite volume and (or) zero temperature.
\end{abstract}

The Witten-Veneziano relation (WVR) [1], 2] plays an important role in identifying topological excitations in gauge theories with fermions. It relates the topological susceptibility of pure gauge theory without fermions to the main fermionic contributions of the complete theory. Since in the general derivation some approximations are involved, it is by educational reasons worthwhile to study models in which the WVR is exact. We choose the Schwinger Model (SM) [3, 4]. However, a correct treatment requires to start with the SM on a compact space, and to consider the infinite space-time limit only at the end.

According to Witten and Veneziano for the SM the following formula

$$
m_{\eta^{\prime}}^{2}=\frac{4}{\left|f_{1}\right|^{2}} \chi_{t o p}^{(0)}
$$

should hold. Here $m_{\eta^{\prime}}$ is a mass of the $U(1)$ pseudoscalar " $\eta^{\prime}$ meson", $f_{1}$ is its decay constant, and $\chi_{\text {top }}^{(0)}$ is a topological susceptibility in pure $U(1)$ theory. For the SM we have $m_{\eta^{\prime}}=m=\frac{e}{\sqrt{\pi}}$ and $f_{1}=\frac{i}{\sqrt{\pi}}$, i.e. the "massive meson" of the SM is identified with $\eta^{\prime}$ particle.

Seiler and Stamatescu [3] reanalyzed the WVR in Euclidean space-time and their final result, applied to the SM, reads

$$
m_{\eta^{\prime}}^{2}=\frac{4}{\left|f_{1}\right|^{2}} P^{(0)}(0)
$$

\footnotetext{
*e-mail address: azhep@lan.ab.az

${ }^{\dagger}$ e-mail address: joos@x4u2.desy.de

‡e-mail address: wipf@tpi.uni-jena.de
} 
The topological susceptibility of pure gauge theory in the WVR has been replaced by the contact term $P^{(0)}(0)$ of the two point function of the topological charge density in the theory with massless fermions. In their work they calculated $P^{(0)}(0)$ and showed that formula (2) really holds.

We calculate in the present work $\chi_{\text {top }}^{(0)}$ and $P^{(0)}(0)$ for the SM on the Euclidean torus, and $\chi_{\text {top }}^{(0)}(0)$ on a circle at finite temperature. Finally, we show that at finite temperature (1) and (2) are correct and that they agree in the limit of infinite volume and (or) zero temperature.

\section{Euclidean path integral approach on the torus}

The topological susceptibility $\chi_{\text {top }}$ in the theory on the two-dimensional Euclidean torus $\mathcal{T}$ (with circumferences $L_{1}, L_{2}$ and volume $V=L_{1} L_{2}$ ) is defined as follows

$$
\chi_{\text {top }}=\int_{\mathcal{T}}\langle q(x) q(0)\rangle d^{2} x
$$

where $q(x)$ is the topological charge density

$$
q(x)=\frac{e}{2 \pi} F_{12}(x) \equiv \frac{e}{2 \pi} E(x)
$$

and

$$
\int q(x) d^{2} x=\frac{e}{2 \pi} \int E(x) d^{2} x=k, \quad k \in \mathbb{Z}
$$

is the integer-valued topological charge or instanton number in the two-dimensional $U(1)$ gauge theory, $E(x)=\partial_{1} A_{2}(x)-\partial_{2} A_{1}(x)$ is the field strength. Here and below the spacetimeintegrals extend over the torus $\mathcal{T}$. From Eqs (3) and (4) we obtain

$$
\chi_{\text {top }}=\frac{e^{2}}{(2 \pi)^{2}} \int\langle E(x) E(0)\rangle d^{2} x .
$$

The expectation values $\langle O(A)\rangle$ of an operator $O(A)$ which depends only on the gauge field $A_{\mu}(x)$ are different in the theory with fermions, e.g. the SM, and in the pure electrodynamics on the torus. After integration with respect to fermions in the SM we have [5, 6, 7, 8]

$$
\langle O(A)\rangle=\frac{1}{Z} \int_{\mathcal{A}_{0}} \mathcal{D} A \operatorname{det} D_{A} O(A) e^{-\frac{1}{2} \int d^{2} x E^{2}(x)},
$$

where $D_{A}$ is a Dirac operator in the external electromagnetic field. As a consequence of the index theorem, there are zero modes in the sectors with instanton number $k \neq 0$ [9]. Therefore the renormalized $\operatorname{det} \not_{A}$ is unequal to zero only in the trivial sector $\mathcal{A}_{0}$ of the gauge field configurations with vanishing topological charge. $Z$ is the partition function in this theory

$$
Z=\int_{\mathcal{A}_{0}} \mathcal{D} A \operatorname{det} \not D_{A} e^{-\frac{1}{2} \int d^{2} x E^{2}(x)}
$$

In pure gauge theory (where expectation values will be denoted by $\langle\cdots\rangle_{0}$ ) all instanton sectors contribute to the expectation value $\langle O(A)\rangle_{0}$ :

$$
\langle O(A)\rangle_{0}=\frac{1}{Z_{g}} \sum_{k \in \mathbb{Z}} \int_{\mathcal{A}_{k}} \mathcal{D} A O(A) e^{-\frac{1}{2} \int d^{2} x E^{2}(x)}
$$


and the partition function reads

$$
Z_{g}=\sum_{k \in Z} \int_{\mathcal{A}_{k}} \mathcal{D} A e^{-\frac{1}{2} \int d^{2} x E^{2}(x)}
$$

In both theories in the sector $\mathcal{A}_{k}$ with topological charge $k$ the gauge potential has the form

$$
A_{\mu}^{(k)}(x)=A_{\mu}^{(0)}(x)+C_{\mu}^{(k)}(x)
$$

where $A_{\mu}^{(0)}(x)$ is a single valued " continuous" function on $\mathcal{T}$ and $C_{\mu}^{(k)}(x)$ is a global instantontype potential which in the Lorentz gauge reads

$$
C_{\mu}^{(k)}(x)=-\frac{\pi k}{e V} \epsilon_{\mu \nu} x_{\nu}
$$

For $A_{\mu}^{(0)}(x)$ we may use the Hodge decomposition

$$
A_{\mu}^{(0)}(x)=\partial_{\mu} a(x)+t_{\mu}+\epsilon_{\mu \nu} \partial_{\nu} b(x),
$$

where $\partial_{\mu} a(x)$ is pure gauge, $t_{\mu}$ is a (constant) toron field restricted to the dual torus $0 \leq t_{\mu} \leq$ $T_{\mu} \equiv 2 \pi / e L_{\mu}, \epsilon_{\mu \nu} \partial_{\nu} b(x)$ is a curl and $a(x)$ and $b(x)$ are continuous on $\mathcal{T}$ and orthogonal to the constant functions: $\int a(x) d^{2} x=\int b(x) d^{2} x=0$ (on the torus the Laplacian $\square \equiv \partial_{1}^{2}+\partial_{2}^{2}$ is invertible only on functions which integrate to zero).

So the path measure in Eqs.(7) - (10) has a form

$$
\int \mathcal{D} A \cdots=\int \mathcal{D} b \int \mathcal{D} a \int_{0}^{T_{1}} d t_{1} \int_{0}^{T_{2}} d t_{2} \cdots
$$

The two-point function $\langle E(x) E(y)\rangle$ has been calculated in the SM on the torus [5, 8] with the following result:

$$
\langle E(x) E(y)\rangle=\delta(x-y)-m^{2} G_{m}(x-y),
$$

where $\delta(x)$ is the $\delta$-function on the torus and

$$
G_{m}(x)=\frac{1}{V} \sum_{n_{1}, n_{2}} \frac{e^{2 \pi i\left(n_{1} x_{1} / L_{1}+n_{2} x_{2} / L_{2}\right)}}{m^{2}+\left(\frac{2 \pi}{L_{1}}\right)^{2} n_{1}^{2}+\left(\frac{2 \pi}{L_{2}}\right)^{2} n_{2}^{2}}
$$

is the Greens function of massive scalars on it. From Eqs.(6) and (15) we see that the contact term is

$$
P^{(0)}(0)=\frac{e^{2}}{4 \pi^{2}}
$$

As is generally true in gauge theory with massless fermions, the topological susceptibility $\chi_{\text {top }}$ vanishes in the SM and therefore the relation (国) holds.

Now let us calculate the two-point function $\langle E(x) E(y)\rangle_{0}$ in pure electrodynamics. Using the decomposition Eq.(13) we get for the field strength

$$
E(x)=-\square b(x)+\frac{2 \pi k}{e V}
$$


and for the action

$$
\frac{1}{2} \int d^{2} x E^{2}(x)=\pi \tau k^{2}+\frac{1}{2} \int d^{2} x b(x) \square^{2} b(x), \quad \tau=2 \pi / e^{2} V .
$$

Then

$$
\begin{aligned}
\langle E(x) E(y)\rangle_{0} & =\left(\frac{2 \pi}{e V}\right)^{2} \frac{\sum k^{2} e^{-\pi \tau k^{2}}}{\sum e^{-\pi \tau k^{2}}}+\frac{\int \mathcal{D} b \square b(x) \square b(y) e^{-\frac{1}{2} \int d^{2} x b(x) \square^{2} b(x)}}{\int \mathcal{D} b e^{-\frac{1}{2} \int d^{2} x b(x) \square^{2} b(x)}} \\
& =\left(\frac{2 \pi}{e V}\right)^{2} \frac{\sum k^{2} e^{-\pi \tau k^{2}}}{\sum e^{-\pi \tau k^{2}}}+\delta(x-y)-\frac{1}{V}
\end{aligned}
$$

where one sums over all $k \in \mathbb{Z}$. The presence of the last term in Eq.(20) is due to the fact that $b(x)$ does not have a zero mode since it integrates to zero. From Eqs(6) and (20) we get for the topological susceptibility in pure electrodynamics

$$
\chi_{\text {top }}^{(0)}=\frac{1}{V} \frac{\sum k^{2} e^{-\pi \tau k^{2}}}{\sum e^{-\pi \tau k^{2}}} .
$$

In pure electrodynamics all instanton sectors contribute to the topological susceptibility. This remains true in infinite volume limit, as we shall see below.

Using the definition of the Jacobi's $\theta_{3}$ function [12]

$$
\theta_{3}(z \mid \tau)=\sum_{n=-\infty}^{n=\infty} e^{-\tau \pi n^{2}+2 \pi i n z}
$$

we may rewrite Eq.(21) in their terms:

$$
\chi_{\text {top }}^{(0)}=-\frac{1}{4 \pi^{2} V} \frac{\theta_{3}^{\prime \prime}(0 \mid \tau)}{\theta_{3}(0 \mid \tau)} .
$$

\section{Topological susceptibility in pure electrodynamics on a circle}

Pure electrodynamics in two dimensions is defined in a non-trivial way only on a compact space where it has non-trivial gauge invariant solutions. Therefore we consider it again on a circle with circumference $L_{1}$. Manton [10] was the first who considered pure electrodynamics on a circle and showed that in this model there is not a unique canonical quantization, because the representation of the electric field operator contains an arbitrary real parameter

$\theta$. The Hamiltonian has eigenvalues: $E_{k}=\frac{1}{2} L_{1} e^{2}(k+\theta / 2 \pi)^{2}, k \in \mathbb{Z}$. In this theory the $\theta$ angle (the fractional part of $\theta / 2 \pi$ ) is a relevant parameter and different values of $\theta$ separate different worlds. It corresponds to the famous $\theta$ angle of $S U(n)$ gauge theories. In contrast, as in the case of quantum chromodynamics with massless fermions [11, in the SM the angle $\theta$ plays non physical role.

The topological susceptibility in this case reads

$$
\chi_{t o p}^{(0)}(0)=\left.\frac{1}{L_{1}} \frac{\partial^{2} F(\theta)}{\partial \theta^{2}}\right|_{\theta=0}
$$


where $F(\theta)=-\frac{1}{\beta} \log Z(\theta)$ is the free energy and $Z(\theta)$ the partition function at temperature $T=1 / \beta$ :

$$
Z(\theta)=\sum_{k} e^{-\beta E_{k}(\theta)}=\sum_{k} e^{-\frac{1}{2} \beta L_{1} e^{2}(k+\theta / 2 \pi)^{2}}
$$

From Eqs.(24) and (25) it follows that

$$
\chi_{\text {top }}^{(0)}(0)=-\frac{1}{L_{1} \beta} \frac{Z^{\prime \prime}(0)}{Z(0)}=\frac{e^{2}}{4 \pi^{2}}\left(1-\beta L_{1} e^{2} \frac{\sum k^{2} e^{-\frac{1}{2} \beta L_{1} e^{2} k^{2}}}{\sum e^{-\frac{1}{2} \beta L_{1} e^{2} k^{2}}}\right) .
$$

Now we can use the following transformation formula [12] between $\theta_{3}$-functions of zero argument

$$
\theta_{3}(0 \mid \tau)=\frac{1}{\tau} \theta_{3}(0 \mid-1 / \tau)
$$

and prove that

$$
\chi_{\text {top }}^{(0)}(0)=\chi_{\text {top }}^{(0)}
$$

if we take $\beta=L_{2}$. Thus the path integral approach and canonical approach give the same result for the topological susceptibility.

A systematic comparison between the Hamiltonian approach for the SM on a circle and the Euclidean path integral approach on the torus was done in the forthcoming paper [13]. There it is shown how to obtain Eq.(15) within the Hamiltonian approach.

\section{The infinite volume limit}

In order to consider the limits of infinite volume $\left(L_{1} \rightarrow \infty\right)$ and/or the zero temperature $\left(L_{2} \rightarrow \infty\right)$ we will use the following expansion [14]:

$$
\frac{\theta_{3}^{\prime \prime}(0 \mid \tau)}{\theta_{3}(0 \mid \tau)}=-\frac{2 \pi}{\tau}-\frac{8 \pi^{2}}{\tau^{2}} e^{-\pi / \tau}\left(1-2 e^{-\pi / \tau}\right)+\cdots
$$

Then from Eq.(23) we find

$$
\chi_{\text {top }}^{(0)}=\frac{e^{2}}{4 \pi^{2}}+\cdots,
$$

where $\cdots$ are terms which disappear if at least one of circumferences $L_{i}$ tends to infinity. Thus we have shown that in this cases the topological susceptibility (23) agrees with the contact term (17).

Acknowledgment: S.A. would like to thank DAAD for a grant which allowed to realize this project. 


\section{References}

[1] E.Witten, Nucl.Phys. B149 (1979) 285.

[2] G.Veneziano, Nucl.Phys. B159 (1979) 213.

[3] E.Seiler and I.O.Stamatescu, preprint MPI-PAE/PTh 10/87, 1987.

[4] C.Gattringer, Ann.Phys. 250 (1996) 389; Ph.D.thesis, MPI-Ph/95-24, hep-th 9503137.

[5] I.Sachs and A.Wipf, Helv.Phys.Acta 65 (1992) 652.

[6] H.Joos and S.Azakov, Helv.Phys.Acta 67 (1994) 723.

[7] I.Sachs and A.Wipf, Phys.Lett. B326 (1994) 105.

[8] S.Azakov, Fortschr.Phys. 45 (1997) 589.

[9] H.Joos, Helv.Phys.Acta 63 (1990) 652.

[10] N.Manton, Ann.Phys.(N.Y.) 159 (1985) 220.

[11] R.Jackiw and C.Rebbi, Phys.Rev.Lett. 37 (1976) 172.

[12] A.Erdélyi (Director), Higher Transcedental Functions, Vol.2, Chapter 13.

[13] S.Azakov and H.Joos, in preparation.

[14] F.Tölke, Praktische Funktionenlehre, Zweiter Band, Springer-Verlag, 1966. 